\title{
Interface
}

\section{PREVALÊNCIA E FATORES DE RISCO PARA DEPRESSÃO PÓS-PARTO ENTRE MÃES RESIDENTES NA ZONA URBANA DE BARBALHA.}

\author{
Rayanne Cunha Pinheiro (1) \\ Antônio Magdo Gonçalves (2) \\ Estelita Pereira Lima (3) \\ Gislene Farias de Oliveira (4)
}

\begin{abstract}
Resumo
A fase puerperal é influenciada por diversos fatores biopsicossociais, caracterizando um momento de grande instabilidade emocional. Isto tende a tornar a mulher mais vulnerável a transtornos psiquiátricos, dentre eles, a Depressão Pós-Parto (DPP). O presente estudo objetivou conhecer a propensão para a DPP entre mães da zona urbana da cidade de BarbalhaCE bem como as variáveis sócio-econômicas que se correlacionam com esta perturbação. Os dados foram coletados através de um questionário sócio-econômico previamente estruturado; do Questionário Avaliativo de Fatores para Depressão Pós-Parto, e da Escala de Edimburgo. A amostra foi aleatória e constituída de 41 mães com filhos de 0 a seis meses de idade, no mês de junho de 2009. A análise dos dados foi feita através do Programa Epi-Info versão 3.3.2. As associações entre as variáveis de interesse foram avaliadas pelo Teste exato de Fisher. Adotou-se o nível de significância de 5\%. Os Resultados eliciaram 46,3\% de sujeitos com sintomatologia de DPP. Houve correlação estatística positiva com o fato de a mãe ser solteira e com o fato da gravidez não ser planejada. Não houve correlação com antecedentes psiquiátricos na família da mãe e uso de bebidas alcoólicas por parte das mesmas. Conclusão: Constatou-se uma alta freqüência de DPP (46,3\%) e ainda uma intrínseca relação desse transtorno psiquiátrico com fatores sociais e de organização e familiar. Assim, infere-se uma notória necessidade de uma maior atenção das autoridades, destinando um maior foco para programas de planejamento familiar.
\end{abstract}

Palavras-Chave: Depressão, Parto, Mães. 


\section{Introdução}

O puerpério caracteriza-se por ser uma nova fase da vida da mulher. Esta fase pode ser influenciada pelos mais diversos fatores biopsicossociais, caracterizando um momento de grande instabilidade emocional e vulnerabilidade a um amplo espectro de transtornos psiquiátricos (SILVA, BOTTI, 2005).

A literatura médica destaca três tipos de manifestações psíquicas no pós-parto: a Disforia do Pós-Parto (Puerperal Blues), a Depressão Pós-Parto e a Psicose Puerperal

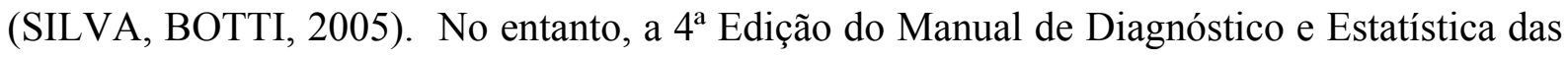
Perturbações Mentais (DSM-IV) e a Décima Revisão Internacional das Doenças (CID-10) não fazem uma distinção dos transtornos de humor no pós-parto dos transtornos de humor de outras fases da vida.

A proposta mais aceita para justificar os transtornos psiquiátricos pós-parto e possivelmente outras síndromes menos severas está embasada na perda da placenta no momento do parto, que é seguida por uma queda acentuada tanto no estrógeno quanto progesterona sérica. Estas mudanças não são diretamente responsáveis por sintomas psicológicos extensos, mas iniciam processos que eventualmente conduzem a sintomas.

Durante os dias imediatamente após o parto, a perfusão diminui na glândula hipófíse, proporcionando uma queda na produção de seus hormônios, um deles, o hormônio adrenocorticotrófico $(\mathrm{ACTH})$, sofre uma redução acentuada em seus níveis séricos o que induz, através de uma alça de feed-back positiva, a uma queda na produção de cortisol (hormônio anti- estresse) por parte da glândula adrenal.

Essa redução do cortisol é percebida por neurônios sensíveis às concentrações do hormônio, de forma que esses neurônios emitem descargas repetidas e extensas quando atingem o limiar mínimo de concentração de cortisol. Essas descargas estimulam os centros autonômicos do hipotálamo provocando ansiedade e topor. A insônia que também é característica, segue a estimulação dos centros do sono adjacentes aos sensores de cortisol. Assim, essas mudanças hormonais e conseqüentemente autonômicas conduzem aos sintomas característicos da Depressão Pós-Parto (KAPLAN, BEJAMIN, 1999).

A Depressão Pós-Parto (DPP) é o transtorno de maior importância no puerpério devido a sua alta prevalência, além da possível geração de implicações negativas na relação da díade mãe-bebê. Assim, devido ao estado afetivo não-responsivo da mãe deprimida há uma 
geração de prejuízos na qualidade da interação dessa díade. Estudos demonstram que o estado afetivo da mãe deprimida altera o seu tipo de vocalização com o bebê (BETTES, 1988), há também um processo de progressivo desligamento através de micro eventos repetidos como o rompimento momentâneo do contato visual com o bebê sem a tentativo de restabelecê-lo logo em seguida (STERN, 1997). Dessa maneira, o bebê tende a diminuir o nível de respostas contingentes à mãe, experimentando um tipo desorganização comportamental, pois o bebê percebe as mínimas alterações no comportamento materno (FIELD, HEALY, GOLDSTEAN, GUTHERTZ, 1990).

A Depressão Pós-Parto é um transtorno psíquico que pode revelar-se de grau modero a severo. Segundo critérios clínicos de diagnóstico do DSM-IV, as manifestações clínicas mais comuns são humor deprimido, anedonia, mudanças significativas no peso ou apetite, insônia ou hipersônia, agitação ou retardo psicomotor, fadiga, sentimentos de inutilidade ou culpa, capacidade diminuída de pensar, de concentrar-se, indecisão, pensamentos recorrentes de morte, tendo de haver a manifestação de pelo menos cinco desses sintomas concomitantemente. Esse transtorno costuma ter início insidioso na $2^{\mathrm{a}}$ a $3^{\mathrm{a}}$ semana do puerpério, tendo de perdurar por pelo menos duas semanas (SUSMAN apud RUSCHI, SUN, MATTAR, FILHO, ZANDONADE, LIMA, 2007). Sendo este um relevante problema da Saúde Pública, pois atinge 19,1\% das puérperas brasileiras (MORAES, PINHEIRO, SILVA, HORTA, SOUSA, FARIA).

O Objetivo do presente estudo foi conhecer a freqüência de risco para Depressão PósParto entre mães da zona urbana da cidade de Barbalha-CE, assim como determinar os principais fatores de risco associados. Para isso foi necessário também: a) estabelecer o perfil socioeconômico das mães residentes na cidade de Barbalha; b) apontar os fatores de maior correlação com a Depressão Pós-Parto, definido que parcela de mães estão mais sujeitas a mesma e; c) comparar os resultados obtidos através da Escala de Edimburgo, com os atuais valores estabelecidos para o Nordeste.

Trata-se de um estudo descritivo do tipo transversal, fundamentado nos dados obtidos através de 41 entrevistas com mães de bebês até 6 meses de nascidos. Foi realizado no município de Barbalha, região sul no Ceará, localizado na Macroregião do Cariri. O município, considerado de pequeno porte, conta com um população de 52.496 habitantes e uma área de 479,184km². O Índice de Desenvolvimento Humano Municipal* é 0,687, sendo considerado um valor de IDH médio.

As entrevistas foram realizadas no próprio domicílio das mães. Assim, foram visitados 39 domicílios. Essas residências visitadas estão distribuídas no espaço urbano da cidade. A 
área urbana da mesma foi dividida virtualmente em quatro quadrantes com a colaboração dos dados da Secretaria de Saúde. Dentro de cada quadrante da cidade, foi sorteado um bairro. Os bairros sorteados foram: Alto do Rosário, Conjunto Nossa Senhora de Fátima, Cirolândia e Centro. Nestes, as visitas foram aleatórias, pois nem sempre nas residências encontrávamos mulheres com o perfil necessário para nossa pesquisa, mães com filhos de até seis meses de idade.

Ao encontrarmos residências com mães que se encaixavam no perfil da pesquisa, as mesmas eram convidadas a participar de nossa pesquisa. Toda uma explanação era feita a respeito do estudo. Após, o Termo de Consentimento Livre e Esclarecido (anexo III) era lido para a mesma e em seguida, se consentida à participação, pesquisador e participante assinavam o termo.

Na primeira fase da entrevista, foi aplicado O Questionário Avaliativo de Fatores para Depressão Pós-Parto (anexo I) contendo 18 perguntas, dessas 16 objetivas e 2 subjetivas. Entre os questionamentos objetivos estão à data de nascimento do bebê, a idade da mãe, o estado civil, a religião, a cor ou raça, a renda familiar mensal, a escolaridade, a gravidez planejada ou não, a quantidade de filhos, a prática da amamentação, o uso de álcool, o uso de tabaco, o histórico de doenças psiquiátricas na família. Entre os questionamentos subjetivos estão a possibilidade hipotética de poder recomeçar e mudar algo e a sensação de estar diferente após a gravidez, caso sim, explicando simplificadamente que tipo de mudança.

Nesta fase inicial de perguntas, as mães não demonstravam qualquer dificuldade de entender ou responder as perguntas objetivas, no caso das perguntas subjetivas houve uma certa dificuldade de expressar em palavras o que desejavam mudar em suas vidas ( pergunta 12) e como se sentiam diferente depois do nascimento do bebê. No entanto, apesar dessa dificuldade, consideramos que a maioria entendeu e respondeu satisfatoriamente esse primeiro bloco de perguntas.

Na segunda fase da entrevista, foi aplicada a Escala de Edimburgh (anexo II), um questionário elaborado para avaliação de Depressão Pós-Parto, que contém dez perguntas. Cada pergunta cotém quatro opções de respostas que são pontuadas de 0 a 3 , de acordo com a presença ou intensidade dos sintomas: humor deprimido ou disfórico, distúrbio do sono, perda do prazer, diminuição do desempenho, culpa e idéias de morte e suicídio (Quadro II),( MATAR, SILVA et al, 2007).

Essa Escala de Depressão Pós-parto de Edinburgh - EPDS (Cox, Chapman et al. 1996), é um instrumento desenhado especificamente para triagem desse transtorno do humor e tem sido amplamente utilizada. No Brasil, há dois estudos de validação bem sucedido de uma 
versão em português. Um dos estudos foi conduzido em Brasília, contudo a amostra foi composta por mulheres de alto nível social e escolar. Isto demandou um estudo de validação no nordeste, onde há um perfil cultural, social e escolar diferente. O outro estudo foi conduzido no Nordeste, tornando-o mais próximo da realidade da cidade de Barbalha onde foi conduzido nosso estudo (CANTILINO, ALBUQUERQUE, et al, 2007). A pontuação da escala é medida da seguinte forma: para os itens 1,2 e 4, a pontuação para a sequência é 0,1,2,3. Para os demais itens, a sequência é 3,2,1,0.

Nessa fase do estudo, pode-se notar uma certa dificuldade das mães de compreender as afirmações e opções da escala. Assim, consideramos o desempenho dessa fase apenas satisfatória.

Após a conclusão da coleta de dados, iniciamos a análise dos dados no Programa EpiInfo versão 3.3.2, na busca de dados relevantes para atingir os objetivos da pesquisa e principalmente de variáveis estatisticamente relacionadas.

\section{Análise dos Dados}

Quanto a idade, 14,6\% das mães entrevistadas são adolescentes de 12 a 18 anos, assim, notamos um alto índice de gravidez na adolescência. Observamos também que mais da metade das mães, 51,2\%, são jovens entre 19 e 25 anos (Gráfico 1).

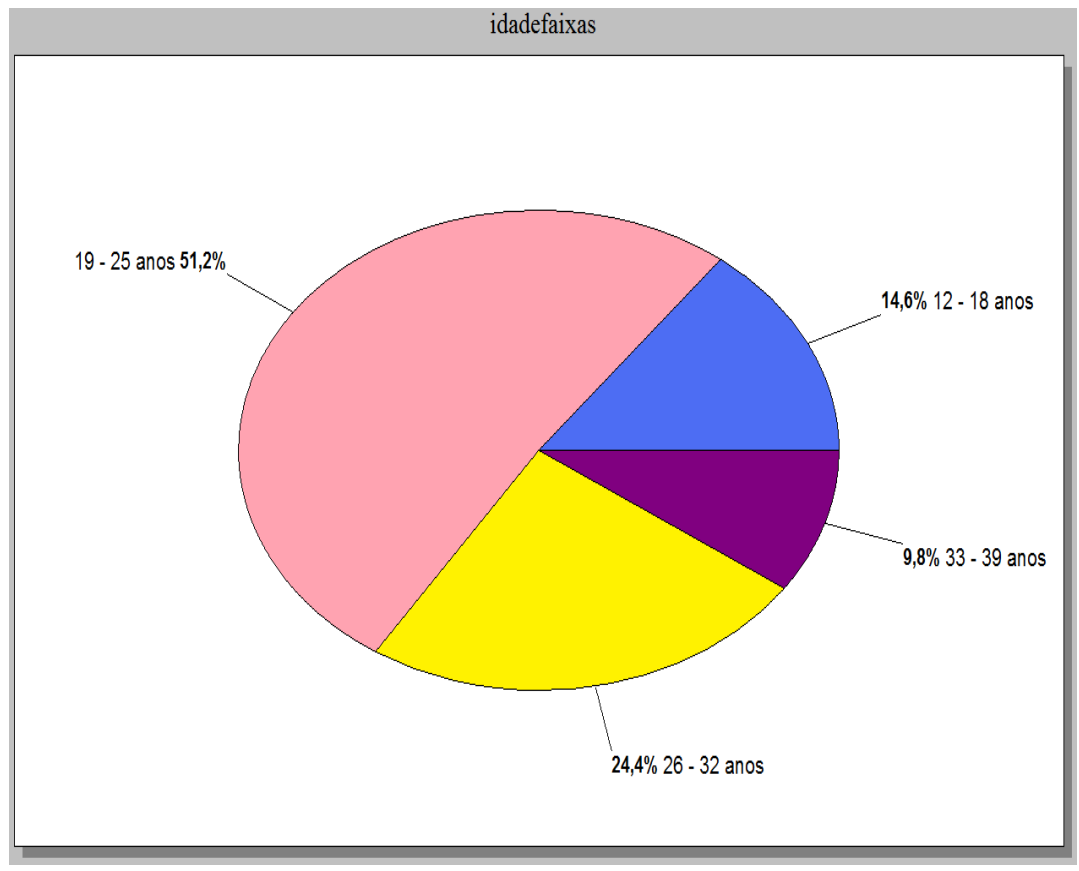

Gráfico I. Faixa de idades das mães entrevistadas. Barbalha, Junho de 2009. 
Com relação a cor ou raça, observou-se que 56\% das entrevistadas declaram como cor ou raça Parda ou Mulata, seguida da segunda maior porcentagem, 39\%, que se declaram brancas. Nenhuma mãe declarou ser amarela ou indígena (Gráfico II).

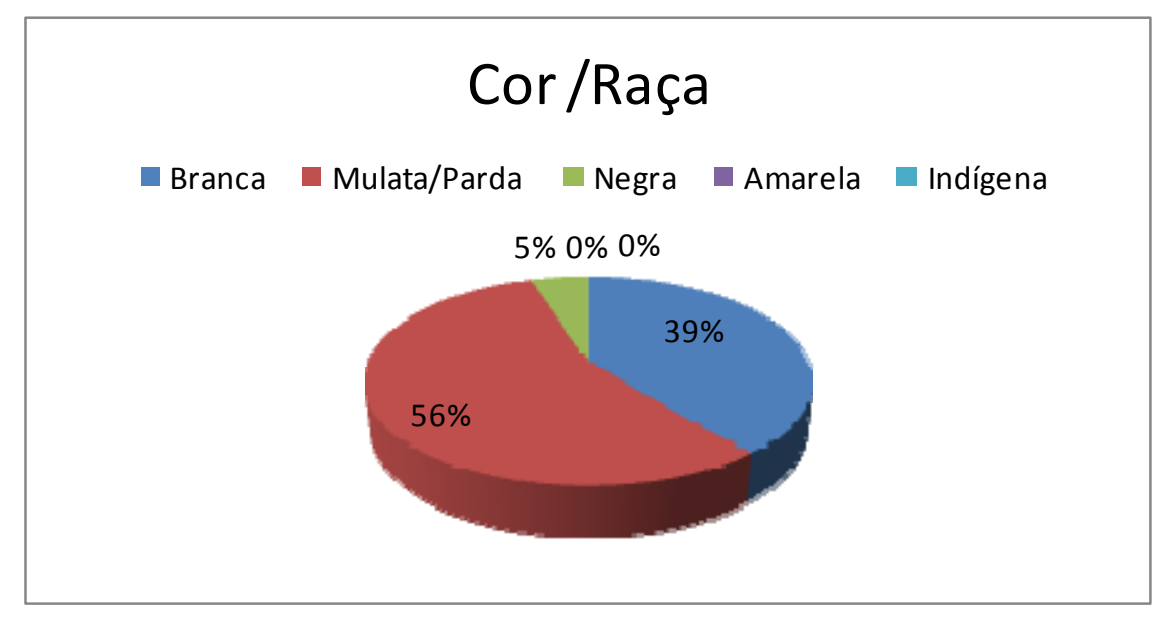

Gráfico II. Cor ou Raça declarada pelas entrevistadas. Barbalha, Junho de 2009

Com relação a religião, 90\% das entrevistadas declaram-se católicas (Gráfico III).

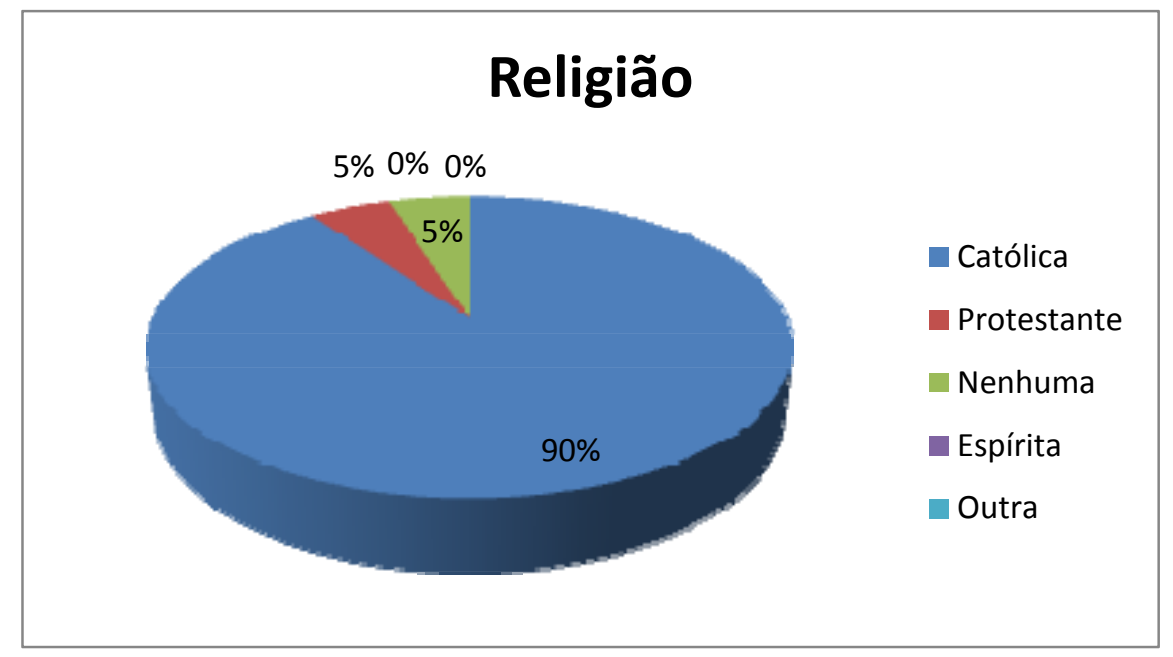

Gráfico III. Religião das entrevistadas. Barbalha, Junho de 2009

Sobre a renda familiar, observou-se que $41,5 \%$ das mães entrevistadas possuíam à época do estudo, uma renda familiar de 232,50 a 465,00 reais, ou seja, de meio até um salário mínimo, caracterizando a baixa renda da população (Tabela 1). 
Tabela 1. Porcentagem de mães com renda familiar de meio até um salário mínimo

\begin{tabular}{|l|l|l|l|l|l|}
\hline De meio até 1 salário & Frequency & Percent & Cum Percent & \\
\hline Yes & 17 & $41,5 \%$ & $41,5 \%$ & \\
\hline No & 24 & $58,5 \%$ & $100,0 \%$ & \\
\hline Total & 41 & $100,0 \%$ & $100,0 \%$ & \\
\hline
\end{tabular}

Também observou-se que apenas 9,8\% das mães entrevistadas possuíam renda familiar acima de 2325,00. Logo, percebe-se a grande desigualdade social na amostra entrevistada (Tabela 2).

Tabela 2. Porcentagem de mães com renda familiar de cinco salários mínimos em diante

\begin{tabular}{|l|l|l|l|l|}
\hline De 5 salários em diante & Frequency & Percent & Cum Percent & \\
\hline Yes & 4 & $9,8 \%$ & $9,8 \%$ & \\
\hline No & 37 & $90,2 \%$ & $100,0 \%$ & \\
\hline Total & 41 & $100,0 \%$ & $100,0 \%$ & \\
\hline
\end{tabular}

Observou-se também informações sobre a amamentação. Os dados estão disposto a seguir.

Tabela 3. Porcentagem de mães que amamentam desde o nascimento

\begin{tabular}{|c|c|c|c|c|c|}
\hline $\begin{array}{l}\text { Amamenta } \\
\text { nascimento? }\end{array}$ & desde $\quad 0$ & Frequency & Percent & \begin{tabular}{|l} 
Cum \\
Percent
\end{tabular} & \\
\hline Yes & & 41 & $100,0 \%$ & $100,0 \%$ & \\
\hline Total & & 41 & $100,0 \%$ & $100,0 \%$ & \\
\hline
\end{tabular}

De acordo com a Tabela 3, 100\% das mães entrevistadas amamentaram seus filhos desde o nascimento. Isso é um provável reflexo do trabalho realizado pelos hospitais locais e pelo Centro Materno infantil, que possuem um trabalho intenso de aconselhamento e acompanhamento Pró-Aleitamento Materno.

Com relação ao histórico familiar de doenças psiquiátricas, observou-se que 36,6\% das entrevistadas alegaram ter, algum parente com algum tipo de transtorno psiquiátrico em suas famílias (Gráfico IV). 


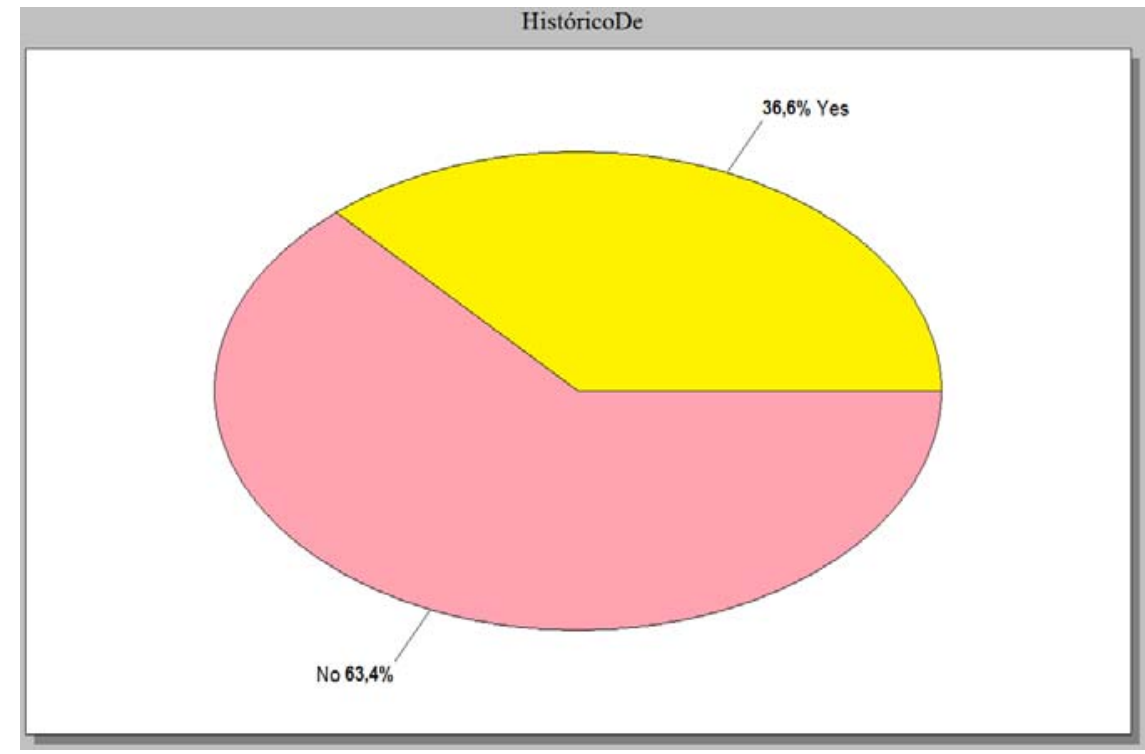

Gráfico IV. Porcentagem de Histórico de Doenças Psiquiátricas na família

\title{
Discussão
}

Tabela 4. Freqüências da Escala de Edimburgo

\begin{tabular}{|l|r|r|r|l|}
\hline EPDS & Frequency & Percent & Cum Percent & \\
\hline EPDS (-) & 22 & $53,7 \%$ & $53,7 \%$ & \\
\hline EPDS (+) & 19 & $46,3 \%$ & $100,0 \%$ & \\
\hline Total & 41 & $100,0 \%$ & $100,0 \%$ & \\
\hline
\end{tabular}

\author{
95\% Conf Limits \\ EPDS (-) 37,4\% 69,3\% \\ EPDS (+) $30,7 \% 62,6 \%$
}

Observou-se que, 46,3\% das entrevistada possuem uma Escala de Edimburgo positiva, ou seja, somam 11 escore ou mais na escala, caracterizando assim a sintomatologia de Depressão Pós- Parto. 
Tabela 5. Relação entre a variável Solteira e a variável Escala de Edimburgo

\begin{tabular}{|r|r|r|r|}
\hline \multicolumn{4}{|c|}{ EPDS } \\
\hline solteira & EPDS (-) & EPDS (+) & TOTAL \\
\hline Yes & 1 & 11 & 12 \\
Row \% & 8,3 & 91,7 & 100,0 \\
Col \% & 4,5 & 57,9 & 29,3 \\
\hline No & 21 & 8 & 29 \\
Row \% & 72,4 & 27,6 & 100,0 \\
Col \% & 95,5 & 42,1 & 70,7 \\
\hline TOTAL & 22 & 19 & 41 \\
Row \% & 53,7 & 46,3 & 100,0 \\
Col \% & 100,0 & 100,0 & 100,0 \\
\hline
\end{tabular}

Fisher exact 0,0002168967

Com relação a variável Solteira e a escala de Edinburgh, observou-se que, sendo a tabela e o valor do Teste de Fisher exato, de valor maior que 5\%, podemos afirmar que as variáveis Solteira - o fato de a puépera ser mãe solteira, e a variável Escala de Edimburgh Positiva, estas estão estatisticamente relacionadas.

Tabela 6. Relação entre a variável Gravidez Planejada e a variável Escala de Edimburgo

\begin{tabular}{|r|r|r|r|}
\hline \multicolumn{4}{|c|}{ EPDS } \\
\hline Gravidez planejada? & EPDS (-) & EPDS (+) & TOTAL \\
\hline Yes & 15 & 4 & 19 \\
Row \% & 78,9 & 21,1 & 100,0 \\
Col \% & 68,2 & 21,1 & 46,3 \\
\hline No & 7 & 15 & 22 \\
Row \% & 31,8 & 68,2 & 100,0 \\
Col \% & 31,8 & 78,9 & 53,7 \\
\hline TOTAL & 22 & 19 & 41 \\
Row \% & 53,7 & 46,3 & 100,0 \\
Col \% & 100,0 & 100,0 & 100,0 \\
\hline
\end{tabular}

Fisher exact 0,0030162843

Quando se correlacionou a variável Gravidez planejada e o Teste de Fisher exato, podemos afirmar que estas se mostraram estatisticamente relacionadas, pois o valor do teste foi 0,0030162843 . 
Tabela 7. Relação entre a variável Histórico de doenças psiquiátricas e a variável Escala de Edimburgo

\begin{tabular}{|r|r|r|r|}
\hline \multicolumn{4}{|c|}{ EPDS } \\
\hline Histórico de doenças psiquiátricas? & EPDS (-) & EPDS (+) & TOTAL \\
\hline Yes & 8 & 7 & 15 \\
Row \% & 53,3 & 46,7 & 100,0 \\
Col \% & 36,4 & 36,8 & 36,6 \\
\hline No & 14 & 12 & 26 \\
Row \% & 53,8 & 46,2 & 100,0 \\
Col \% & 63,6 & 63,2 & 63,4 \\
\hline TOTAL & 22 & 19 & 41 \\
Row \% & 53,7 & 46,3 & 100,0 \\
Col \% & 100,0 & 100,0 & 100,0 \\
\hline
\end{tabular}

Fisher exact $\quad 0,6142758782$

Ao se tomar em consideração o Teste de Fisher exato e a variável Doenças psiquiátricas na família, podemos afirmar que a variável Histórico de doenças psiquiátricas e a Escala de Edimburgo estas não se mostraram estatisticamente relacionadas, pois o valor do teste foi 0,6142758782 .

Tabela 8. Relação entre a variável Uso de álcool e a variável Escala de Edimburgo

\begin{tabular}{|r|r|r|r|}
\hline \multicolumn{4}{|c|}{ EPDS } \\
\hline Uso de álcool? & EPDS (-) & EPDS (+) & TOTAL \\
\hline Yes & 6 & 5 & 11 \\
Row \% & 54,5 & 45,5 & 100,0 \\
Col \% & 27,3 & 26,3 & 26,8 \\
\hline No & 16 & 14 & 30 \\
Row \% & 53,3 & 46,7 & 100,0 \\
Col \% & 72,7 & 73,7 & 73,2 \\
\hline TOTAL & 22 & 19 & 41 \\
Row \% & 53,7 & 46,3 & 100,0 \\
Col \% & 100,0 & 100,0 & 100,0 \\
\hline
\end{tabular}

Fisher exact $\quad 0,6129322599$

Com relação a variável Uso de álcool e a Escala de Edimburgh, estas não se mostraram estatisticamente relacionadas, pois o valor do Teste de Fisher exato foi de 0,6129322599 . 
Tabela 9. Relação entre Número de Filhos e a Escala de Edimburgo

\begin{tabular}{|c|c|c|c|}
\hline \multicolumn{4}{|c|}{ EPDS } \\
\hline Quantos filhos? & EPDS (-) & EPDS (+) & TOTAL \\
\hline \begin{tabular}{|l} 
\\
\end{tabular} & 14 & 12 & 26 \\
\hline Row \% & 53,8 & 46,2 & 100,0 \\
\hline $\mathrm{Col} \%$ & 63,6 & 63,2 & 63,4 \\
\hline 2 & 3 & 4 & 7 \\
\hline Row \% & 42,9 & 57,1 & 100,0 \\
\hline $\mathrm{Col} \%$ & 13,6 & 21,1 & 17,1 \\
\hline 3 & 3 & 0 & 3 \\
\hline Row \% & 100,0 & 0,0 & 100,0 \\
\hline $\mathrm{Col} \%$ & 13,6 & 0,0 & 7,3 \\
\hline 4 & 1 & 0 & 1 \\
\hline Row \% & 100,0 & 0,0 & 100,0 \\
\hline $\mathrm{Col} \%$ & 4,5 & 0,0 & 2,4 \\
\hline 5 & 1 & 2 & 3 \\
\hline Row \% & 33,3 & 66,7 & 100,0 \\
\hline $\mathrm{Col} \%$ & 4,5 & 10,5 & 7,3 \\
\hline 6 & 0 & 1 & 1 \\
\hline Row \% & 0,0 & 100,0 & 100,0 \\
\hline $\mathrm{Col} \%$ & 0,0 & 5,3 & 2,4 \\
\hline TOTAL & 22 & 19 & 41 \\
\hline Row \% & 53,7 & 46,3 & 100,0 \\
\hline $\mathrm{Col} \%$ & 100,0 & 100,0 & 100,0 \\
\hline
\end{tabular}

Descriptive Statistics for Each Value of Crosstab Variable

Obs Total Mean Variance Std Dev

EPDS (-) $22 \quad 38,00001,7273 \quad 1,3506 \quad 1,1622$

EPDS (+) $1936,0000 \quad 1,8947 \quad 2,5439 \quad 1,5949$

Com relação a Média de filhos das puérperas, que possuem a Escala de Edimburgh Positiva é 1,8947 filhos.

Observou-se ainda que a variável Insônia e a variável Escala de Edimburgo estão estatisticamente relacionadas pois o valor do Teste de Fisher exato foi de 0,0000008367 (Tabela 10).

Tabela 10. Relação entre a variável Insônia e a variável Escala de Edimburgo

\begin{tabular}{|r|r|r|r|}
\hline \multicolumn{4}{|c|}{ EPDS } \\
\hline Insônia & EPDS (-) & EPDS (+) & TOTAL \\
\hline Yes & 1 & 15 & 16 \\
Row \% & 6,3 & 93,8 & 100,0 \\
Col \% & 4,5 & 78,9 & 39,0 \\
\hline No & 21 & 4 & 25 \\
Row \% & 84,0 & 16,0 & 100,0 \\
Col \% & 95,5 & 21,1 & 61,0 \\
\hline TOTAL & 22 & 19 & 41 \\
Row \% & 53,7 & 46,3 & 100,0 \\
Col \% & 100,0 & 100,0 & 100,0 \\
\hline
\end{tabular}

Fisher exact 0,0000008367 
Tabela 11. Relação entra a Variável Idéia de Suicídio e a variável Escala de Edimburgo

\begin{tabular}{|r|r|r|r|}
\hline \multicolumn{4}{|c|}{ EPDS } \\
\hline Idéia de suicídio & EPDS (-) & EPDS (+) & TOTAL \\
\hline Yes & 2 & 7 & 9 \\
Row \% & 22,2 & 77,8 & 100,0 \\
Col \% & 9,1 & 36,8 & 22,0 \\
\hline No & 20 & 12 & 32 \\
Row \% & 62,5 & 37,5 & 100,0 \\
Col \% & 90,9 & 63,2 & 78,0 \\
\hline TOTAL & 22 & 19 & 41 \\
Row \% & 53,7 & 46,3 & 100,0 \\
Col \% & 100,0 & 100,0 & 100,0 \\
\hline
\end{tabular}

Fisher exact 0,0382333553

Tomando-se em consideração o Teste de Fisher exato, podemos afirmar que a variável Idéia de Suicídio e a variável Escala de Edimburgo estão estatisticamente relacionadas pois o valor do teste foi 0,0382333553 .

\section{Conclusões}

Entre as mães investigada, 46,3\% apresentaram uma pontuação igual ou acima de 11 pontos na Escala de Edimburgo, caracterizando a sintomatologia de Depressão Pós-Parto. Essa alta freqüência encontra-se bastante acima se compara aos estudos de validação da EPDS realizados no Brasil (CANTILINO, ALBUQUERQUE, et AL, 2007) e (SANTOS, MARTINS, PASQUALI, 1999).

Através da análise realizada pelo Programa Epi-Info, descartou-se relações estatísticas entre as variáveis Histórico de Doenças Psiquiátricas na Família e Uso de Álcool com a Escala de Edimburgo Positiva. Logo, conclui-se que o fato de a mãe possuir familiares com antecedentes psiquiátricos ou fazer o uso de bebida alcoolica e não constituem um fatores predisponentes estatisticamente significantes para Depressão Pós-Parto.

Correlacionando o fato de a puérpera ser mãe solteira e a Escala de Edimburgo, podemos concluir que a relação entre essas variáveis é estatisticamente significante. Ou seja, o fato de a mães ser solteira constitui um fator predisponente para a Depressão Pós-Parto.

Correlacionando agora a variável Gravidez Planejada com a Escala de Edimburgo novamente encontramos uma relação estatisticamente significante. Logo, podemos deduzir que o fato de a gravidez acontecer de forma inesperada ou não planejada caracteriza um fator de risco para a Depressão Pós-Parto. 
Analisando a relação entre a Escala de Edimburgh e o número de filhos das mães entrevistadas podemos perceber que a média de filhos das Mães com EPDS (-) é 1,7273 e a média das mães EPDS (+) é 1,8947. Assim, mesmo a média de filhos das mães com a escala positiva para Depressão Pós-Parto ser maior, a diferença não é tão significativa, logo o número de filhos não se mostrou um fator predisponente significante.

Observando os dados das mães que alegam Insônia em maior ou menor grau e os relacionando com a Escala de Edimburgh, podemos notar que a Insônia é uma característica estatisticamente associada a Depressão Pós-Parto, assim como a Idéia de Suicídio com maior ou menor freqüência.

Podemos concluir que os inúmeros estudos epidemiológicos sobre depressão pós-parto confirmam a complexidade do tema ao demonstrar divergência nas prevalências encontradas, multiplicidade dos fatores de risco envolvidos e de etiologias propostas. O grande número de mulheres com sintomas depressivos em nossa amostra confirma que a depressão é problema sério de saúde pública, o que justifica a capacitação dos profissionais de saúde para o reconhecimento precoce da depressão pós-parto, tendo como auxílio instrumentos como a EPDS, pela sua eficácia e praticidade.

\section{Anexo:}

\section{Escala de Edimburgh}

Instruções. Você teve há pouco tempo um bebê e nós gostaríamos de saber como você está se sentindo. Por favor, marque a resposta que mais se aproxima do que você tem sentido NOS ÚLTIMOS SETE DIAS, não apenas como você está se sentindo hoje.

Aqui está um exemplo já preenchido:

\section{Eu tenho me sentido feliz:}

$\square$ Sim, todo o tempo.

凶Sim, na maior parte do tempo.

$\square$ Não, nem sempre.

$\square$ Não, em nenhum momento.

Esta resposta quer dizer: "Eu me senti feliz na maior parte do tempo" na última semana.

Por favor, assinale as questões seguintes do mesmo modo. 


\section{Nos últimos sete dias:}

1. Eu tenho sido capaz de rir e achar graça das coisas.

$\square$ Como eu sempre fiz.

$\square$ Não tanto quanto antes.

$\square$ Sem dúvida menos que antes.

$\square$ De jeito nenhum.

2. Eu sinto prazer quando penso no que está por acontecer em meu dia-a-dia.

$\square$ Como sempre senti.

$\square$ Talvez menos do que antes.

$\square$ Com certeza menos.

$\square$ De jeito nenhum.

3. Eu tenho me culpado sem necessidade quando as coisas saem erradas.

$\square$ Sim, na maioria das vezes.

$\square$ Sim, algumas vezes.

$\square$ Não muitas vezes.

$\square$ Não, nenhuma vez.

4. Eu tenho me sentido ansiosa ou preocupada sem uma boa razão.

$\square$ Não, de maneira alguma.

$\square$ Pouquíssimas vezes.

$\square$ Sim, alguma às vezes.

$\square$ Sim, muitas vezes.

5. Eu tenho me sentido assustada ou em pânico sem um bom motivo.

$\square$ Sim, muitas vezes.

$\square$ Sim, algumas vezes.

$\square$ Não, muitas vezes.

$\square$ Não, nenhuma vez. 
6. Eu tenho me sentido esmagada pelas tarefas e acontecimentos do meu dia-a-dia.

$\square$ Sim. Na maioria das vezes eu não consigo lidar bem com eles.

$\square$ Sim. Algumas vezes não consigo lidar bem como antes.

$\square$ Não. Na maioria das vezes consigo lidar bem com eles.

$\square$ Não. Eu consigo lidar com eles tão bem quanto antes.

7. Eu tenho me sentido tão infeliz que tenho tido dificuldade de dormir.

$\square$ Sim, na maioria das vezes.

$\square$ Sim, algumas vezes.

$\square$ Não, muitas vezes.

$\square$ Não, nenhuma vez.

8. Eu tenho me sentido triste ou arrasada.

$\square$ Sim, na maioria das vezes.

$\square$ Sim, muitas vezes.

$\square$ Não muitas vezes.

$\square$ Não, de jeito nenhum.

9. Eu tenho me sentido tão infeliz que tenho chorado.

$\square$ Sim, quase todo o tempo.

$\square$ Sim, muitas vezes.

$\square$ De vez em quando.

$\square$ Não, nenhuma vez.

10. A idéia de fazer mal a mim mesma passou por minha cabeça.

$\square$ Sim, muitas vezes, ultimamente.

$\square$ Algumas vezes nos últimos dias.

$\square$ Pouquíssimas vezes, ultimamente

$\square$ Nenhuma vez.

\section{Escala de Edimburgo}

J. L. Cox, J. M. Holden, R. Sagovsky, 1987

(C) British Journal of Psychiatry

tradução: Maria Fátima S. dos Santos e Célia C. Moraes

supervisão de tradução: Prof. João Dino F. P. Santos

Depto. de Letras_UnB

validação: Maria Fátima S. Santos, Francisco M. C. Martins e Luis Pasquali _ Depto. de Psicologia _ UnB (PC=12) 


\section{Referências}

SILVA, Elda Terezinha da; BOTTI, Nadja Cristiane Lappann. Depressão puerperal - uma revisão de literatura. Goiânia. UFG, 2005.

RUSCHI, Gustavo Enrico Cabral; SUN, Sue Yazaki; MATTAR, Rosiane; FILHO, Antônio Chambô; ZANDONADE, Eliana; LIMA, Valmir José de. Aspectos epidemiológicos da depressão pós-parto em amostra brasileira.Vitória.UFES, 2007.

MORAES, Inácia Gomes da Silva; PINHEIRO, Ricardo Tavares; SILVA Ricardo Azevedo da; HORTA, Bernardo Lessa; SOUSA, Augusto Duarte Faria. Prevalência da depressão pós-parto e fatores associados. Pelotas. UFP, 2006.

CANTILINO, Amaury ; CARVALHO, João Alberto ; MAIA, A. ; ALBUQUERQUE, C. ; CANTILINO, G. ; SOUGEY, Everton . Validação da Escala de Depressão Pós-Parto de Edimburgo no Nordeste do Brasil. Recife. UFPE, 2007.

COX, J. L., G. CHAPMAN, et al. (1996). "Validation of the Edinburgh Postnatal Depression Scale (EPDS) in non-postnatal women." J Affect Disord 39(3): 185-9.

SANTOS, Maria de Fátima S. dos; MARTINS, C. Francisco; PASQUALI, Luiz. Escalas de auto-avaliação de depressão pós-parto: estudo no Brasil. Brasília. UnB, 2007.

Sobre os autores:

(1) Rayanne Cunha Pinheiro é Acadêmica do Curso de Medicina da Universidade Federal do Ceará. E-mail: r4y4nne@hotmail.com;

(2) Antônio Magdo Gonçalves é Acadêmico do Curso de Medicina da Universidade Federal do Ceará;

(3) Estelita Pereira Lima é Professora do Curso de Medicina da Universidade Federal do Ceará - UFC. E-mail: estelitaplima@hotmail.com;

(4) Gislene Farias de Oliveira é Professora da Universidade Regional do Cariri e do Curso de Medicina da Universidade Federal do Ceará - UFC.

E-mail: gislenefarias@gmail.com

Como citar este artigo (Formato ISO):

PINHEIRO, Rayanne C.; GONÇALVES, Antônio M.; LIMA, Estelita P. e OLIVEIRA, Gislene F. Prevalência e fatores de risco para depressão pós-parto entre mães residentes na zona urbana de Barbalha. Id on Line Revista de Psicologia - Edição Especial Educação e Saúde, set. 2009, vol.1, no.9, p.29-44. ISSN 1981-1189. 